\title{
D'Alembert and the Wave Equation: Its Disputes and Controversies
}

\author{
Agamenon R. E. Oliveira \\ Polytechnic School of Rio de Janeiro, Rio de Janeiro, Brazil \\ Email: agamenon.oliveira@globo.com
}

How to cite this paper: Oliveira, A. R. E. (2020). D'Alembert and the Wave Equation: Its Disputes and Controversies. $A d$ vances in Historical Studies, 9, 229-239. https://doi.org/10.4236/ahs.2020.94019

Received: October 1, 2020

Accepted: November 27, 2020

Published: November 30, 2020

Copyright $\odot 2020$ by author(s) and Scientific Research Publishing Inc. This work is licensed under the Creative Commons Attribution International License (CC BY 4.0).

http://creativecommons.org/licenses/by/4.0/

\begin{abstract}
The solution of the vibrating string problem is one of d'Alembert's fundamental contributions to mathematics and physics. This problem, which attracted the attention of many mathematicians, was solved in his general formulation of the wave equation. Even nowadays, with so much technological progress in computer sciences and experimental methods, there are important applications of wave equation: for example, guyed cables for structural purposes, vibrations in transmission lines and risers of offshore structures, guyed bridges, etc. It remains a very useful tool for these engineering problems.
\end{abstract}

\section{Keywords}

History of Mathematics, History of Mechanical Vibrations, Partial Differential Equations, History of Engineering

\section{Introduction}

The origin and the precise treatment of the vibrations of continuous systems can be found in the discovery of the basic law of elasticity by Hooke (1635-1703) in 1660, in the Newton's second law of motion (1687) and in the principles of differential calculus by Leibniz (1646-1717). Newton's second law is currently used to derive the mathematical model of the vibrating system.

We can begin to briefly describe the history of the wave equation starting with Johann Bernoulli (1667-1748) when he solved the problem of the catenary which had been proposed by his brother Jacob, in 1691. Following these contributions, we emphasize the work of Brook Taylor (1685-1731) who presented in 1715 in his book Methodus incrementorum directa et inversa a discussion on vibrating strings, an interest which certainly appeared from music. Daniel Bernoulli (1700-1782), while living in St. Petersburg (1727-1733), studied the mechanics of 
elastic bodies. He calculated the shape that an elastic line can assume under the action of forces. In this case, Bernoulli defined the natural frequencies and the vibration nodes of strings and showed that the motion of strings is composed of an infinite number of superposed harmonic vibrations (Bernoulli, 1740).

Euler (1707-1783) also examined free vibrations of an elastic string without mass (Euler, 1749). In connection with the development of a solution of vibration string d'Alembert (1717-1783) developed his integration method for a system of linear differential equations. In this context, Daniel Bernoulli established his famous theorem that the solution for the free vibration of strings can be described by a trigonometrical series. The discussion between Euler, d'Alembert, and Daniel Bernoulli ran over a few decades, as we will see below.

Later, Lagrange (1736-1813), showed how one can obtain a solution for that problem. He used a discrete mass model for string vibration, consisting of $n$ masses joined by weightless strings. He solved the resulting system of $n+1$ differential equations where $n$ tends to infinity to obtain the same functional solution as Euler did. Only in 1822 did Fourier (1768-1830) completely solve the problem in his "The Analytical Theory of Heat", in which he proposed that any function could be written as an infinite sum of the sine and cosine trigonometric functions whose periods are integral divisors of the period of the original function (Fourier, 1822).

\section{D’Alembert's Biographical Note}

D'Alembert was born on November 17, 1717, in Paris, and died on October 29, 1783, also in Paris. He was abandoned by his parents on the steps of Saint Jean le Rond church, and adopted by an artisan family (Paty, 1993). Despite this, he received a good education with the support of his biological father, Chevalier Destouches-Canon. With multiple influences on his education such as Jansenism, Malebranchism, and Cartesianism, mathematics drew his attention since the beginning.

He made his acquaintance with the new analysis and the calculus, studying Bernoulli's work and Newtonian science. In 1739, he submitted his first work to the Academy of Science, followed by many others justifying his entrance to the Academy in 1741 as an "Associate adjunct astronomer". Since the 1760's he held a key position in the Academy of Sciences.

He went through a period of almost twenty years of very intense work, culminating with the publication in 1743 of his Traité de dynamique, meaning the general theorem of dynamics or d'Alembert's principle, a kind of unification of mechanics, publishing Traité d'équilibre et du movement des fluids the following year, and in 1752 Essay d une nouvelle theorie des resistances des fluides, in which hydrodynamics became a branch of mechanics. In 1745, he tackled the three-body problem within Newtonian gravitational theory. In 1749, Recherches sur la precession des equinoxes appeared, followed by Recherches sur points importants du systéme du monde, in 1754. 
With all these works d'Alembert, Euler, and Clairaut before Lagrange (1736-1813) and Laplace (1749-1827), became the successors and followers of Newton in the eighteenth century in mechanics and astronomy (Paty, 2002).

In 1747, in mathematics, d'Alembert created and developed a new branch of differential calculus, partial differential calculus, when he postulated a general solution for the wave Equation (Paty, 2002: pp. 237-258).

In 1746, in his Reflexions sur la cause general des vents (1746-1747), he presented the first demonstration of the fundamental theorem of algebra, meaning that an algebraic equation of $\mathrm{n}$ degree has $\mathrm{n}$ possible roots real or imaginary.

\section{D'Alembert's Solution of the Vibrating String Problem}

The concept of partial differential equation was introduced by d'Alembert in about 1740 to solve problems of continuous media. He recognized in this field a new area of differential and integral calculus.

The first partial differential equation appeared in 1743 in his "Traité de Dynamique" and refers to oscillations of a suspended string, as follows (D'Alembert, 1743):

$$
-\mathrm{dd} y=\left[\mathrm{d} y / \mathrm{d} s-(l-s) \mathrm{dd} y / \mathrm{d} s^{2}\right] \mathrm{d} t^{2}
$$

where $s$ is the horizontal displacement, $l$ the string length, $t$ the time, and $y$ the vertical displacement at time $t$ from the equilibrium position, $d$ represents differential operations with respect to time $t$ with $s$ constant.

The first integration of Equation (1) is found in the third part of "Réflexions sur la cause génerale des vents", published by d'Alembert in 1747 . He tried to integrate two differentials in the form $\alpha \mathrm{d} s+\beta \mathrm{d} u$ and $\rho \alpha \mathrm{d} u+\nu \beta \mathrm{d} s+A \mathrm{~d} u+\Psi \mathrm{d} s$, where $A$ and $\Psi$ are given functions of $s$ and $u, \rho$ and $v$ are constants, and $\alpha$ and $\beta$ unknown functions to be determined, such that the two forms are the total differential of functions $z$ and $v$, of $u$ and $s$.

D'Alembert used the integrability conditions proposed by Euler in 1740, as follows:

$$
\mathrm{d} \alpha / \mathrm{d} u=\mathrm{d} \beta / \mathrm{d} s \text { and } v \mathrm{~d} \beta / \mathrm{d} u=\rho \mathrm{d} \alpha / \mathrm{d} s+\varphi
$$

where $\varphi=\mathrm{d} A / \mathrm{d} s-\mathrm{d} \psi / \mathrm{d} u$ is a known function. Using this approach, d'Alembert tries to arrive at a first order system of two partial differential equations with respect to the two unknown functions of $\alpha$ and $\beta$. The model (1) can be rewritten for small vibrations in the form:

$$
\mathrm{d}^{2} y / \mathrm{d} s^{2}=\mathrm{d}^{2} y / \mathrm{d} t^{2}
$$

The units are chosen such that the coefficient constant is equal to one.

Assuming that $y=\varphi(t, s)$ and putting $\mathrm{d} \varphi=p \mathrm{~d} t+q \mathrm{~d} s, \quad \mathrm{~d} p=\alpha \mathrm{d} t+v \mathrm{~d} s$ and $\mathrm{d} q=\mathrm{vd} t+\beta \mathrm{d} s, \quad \alpha=\beta$, then

$$
\begin{aligned}
& \mathrm{d}(p+q)=(\alpha+v) \mathrm{d}(t+s) \\
& \mathrm{d}(p-q)=(\alpha-v) \mathrm{d}(t-s)
\end{aligned}
$$

$\alpha \pm v$ must be function of $t \pm s$ 


$$
\begin{aligned}
& p+q=f(t+s) \\
& p-q=g(t-s)
\end{aligned}
$$

where $f$ and $g$ are arbitrary functions. Then, one has the following relations:

$$
q=1 / 2\{f(t+s)-g(t-s)\}
$$

One further integration gives:

$$
y=\varphi(t+s)+\psi(t-s)
$$

with two arbitrary functions $\varphi$ and $\Psi$.

Introducing boundary conditions in (2), one has:

$s=0$ and $s=a$ for fixed conditions. Thus, $\Psi(t)=-\varphi(t)$ and $\varphi(t+a)=\varphi(t-a)$.

The two arbitrary functions become only one as $\varphi$, which must be periodic with period $2 a$.

The same solution obtained by d'Alembert was achieved by Brook Taylor in 1747. In this case, $y$ is the product of a sinusoidal function of $s$ by a sinusoidal function of $t$.

Euler used the findings of d'Alembert and remarks that the arbitrary functions $\varphi$ and $\Psi$ are determined by the initial conditions: $\varphi(s)+\Psi(-s)=\varphi(s)-\varphi(-s)$ which represents the initial form of the curve, while $\varphi^{\prime}(s)+\Psi^{\prime}(-s)=\varphi^{\prime}(s)-\varphi^{\prime}(-s)$ represents the initial velocities.

After these developments, d'Alembert returned to Brook Taylor's solution by introducing the method of separation of variables in a partial differential equation. He made the separation of variables in the solution and not in the equation, so that he wrote (D'Alembert, 1750):

$$
\varphi(t+s)-\varphi(t-s)=\Delta(t) \psi(s)
$$

introducing the functions $\Delta$ and $\Psi$ of $t$ and $s$, respectively. If the equations of motion are used, $\Delta$ and $\Psi$ must be sinusoidal functions.

Daniel Bernoulli came to debate the string vibration solution in 1753 stating that all kinds of possible motion are simple mode superposition, similar to what Brook Taylor proposed, but with any number of modes. In other words, the solution proposed by Euler as a particular case actually a general solution in a form of a trigonometric series.

In 1767, Euler stated that arbitrary functions which appear in partial differential equations must be general functions and cannot be defined by analytic formulas. This point of view became dominant among mathematicians. Laplace, in 1779 , solved the vibrating string problem with another approach, using finite differences which correspond to a discrete problem.

In the context of the history of differential and integral calculus, d'Alembert is also responsible for the method of separation of variables which would be developed by Fourier in his "The Analytical Theory of Heat". Thus, d'Alembert tried a systematization of this new theory independent of physical applications. 


\section{Euler's Short Intellectual Biography}

Leonhard Euler is undoubtedly the most important mathematician of the eighteenth century. He was born in the vicinity of Basil, in northern Switzerland near the French border, on April 15, 1707, the son of Paul Euler and Margaret Bruchner. After first being taught by his father, Euler went to university at the age of thirteen, where he demonstrated a rare aptitude for mathematics. In addition, he studied with Jean Bernoulli and was directly influenced by Leibniz and Descartes (1596-1650), mainly in mathematics and philosophy. He received mechanical theory from Newton (1642-1727) mainly in mathematics and philosophy (Oliveira, 2007).

He adopted and used Leibniz's mathematical theory for finite and infinitesimal quantities, as well as adopting Newton's concept of force, but diverged with the latter in relation to the idea of absolute space.

At seventeen he wrote a paper on the "Physical demonstration of sound". This is the first noteworthy work of Euler which had a significant influence on research into acoustics.

When Euler was twenty-one, he was nominated by Daniel Bernoulli to the St. Petersburg Academy of Sciences. In 1733 he succeeded him as mathematician in that Academy. Here he improved integral calculus, developed the theory of trigonometric and logarithm functions, and worked hard to simplify analytical expressions in mathematics.

Probably because of his intense intellectual effort, Euler became partially blind in 1735. In 1741 Frederick the Great invited him to the Berlin Academy. He left Russia and stayed in Berlin for 25 years. During this period Euler produced a significant amount of scientific work.

Euler and d'Alembert died in a truly short time between each other, in the fall of 1783. Their deaths marked the end of an eighteenth-century era. Euler was one of the most productive and brilliant mathematicians of all time, with pioneering work in many fields of pure and applied mathematics, mainly in differential and integral calculus and its applications in mechanics and engineering. D'Alembert, in addition to being a great mathematician, can also be considered a philosopher, having earned a great reputation in science, especially in the field of analytical mechanics and in celestial mechanics, the two are similar.

The correspondence between both attests that they both admired and respected each other as brothers. In 1747, Euler wrote: “d'Alembert's treatise on the movements of the moon is certainly of the greatest depth" (Euler, 1980: p. 266). Euler also recognized that d'Alembert had been more successful with planetary movements in a resistant medium than he was. D'Alembert, on the other hand, returns the praiseworthy comments by saying: "No one is deeper and more competent than Euler" (Euler, 1980: p. 288).

This friendly behavior does not exclude their long disputes over the logarithms of negative numbers or the theory of the vibrating string as we will see below. In 1757 , this competition led to a rivalry separating them for a time. In 
1763, the old friendship was finally restored (Knobloch, 2018).

Euler's main works:

- Dissertatio physica de song (Basel, 1727).

- Mechanica sive motus scientia analytice exposita (St. Petersburg, 1736, in 2 Vols.).

- Ennleitung in die arithmetik (1738, in 2 Vols.).

- Tentamen novae theoriae musicae (1739).

- Methodus inveniendi lineas curves maximi minimine proprictate gaudentes (Lausanne, 1744)

- Theoria motuum planetarum et cometarum (Berlin, 1744).

- Opuscula varii argumenti (1745-1751, in 3 Vols.).

- Novae et carrectae tabulae ad loco lunae computanda (1746).

- Tabulae astronomicae solis et lunae (ibid).

- Introductio it analysin infinitorum (Lausanne, 1746, in 2 Vols.).

- Scientia navalis seu tractatus de construendis ac dirigendis navi bus (St. Petersburg, 1749, in 2 Vols.).

- Theoria motus lunae (Berlin, 1753).

- Dissertatio de principio miniminae actionis uma cum examine objectionum cl. Prof. Koenigii (1753).

- Institutiones calculi differentialis cum ejus usu in analysi intuitorum ac doctrina serierum (1755).

- Constructio lentium objectivarum (St. Petersburg, 1762).

- Theoria motus corporum solidorum seu rigidorum (1765).

- Institutiones calculi integralis (St. Petersburg, 1768-1770, in 3 Vols.).

- Lettres à une princesse d'Allemagne sur quelques sujets de physique et de philosophie (St. Petersburg, 1768-1772, in 3 Vols.).

- Introduction to algebra (1770).

- Dioptrica (1767-1771, in 3 Vols.).

- Opuscula analytica (St. Petersburg, 1783-1785, in 2 Vols.).

\section{The Controversy between D'Alembert and Euler}

D'Alembert's work was communicated to the Berlin Academy in 1747 (D'Alembert, 1747). It created a great controversy between him and Euler. This intellectual dispute was over the nature of arbitrary functions which appear in the integration of partial differential equations. As mentioned above, the solution proposed by d'Alembert is given by the arbitrary functions in $y=\varphi(t+s)+\psi(t-s)$. In order to satisfy the extremity conditions: in $s=0$ and $s=a$ the string is fixed, which implies $\varphi(s)=-\psi(t)$ as well as $\varphi(t+a)=\varphi(t-a)$, such that the two arbitrary functions become one, $\varphi$, which must be periodic with a period $2 a$.

Euler criticized d'Alembert's work pointing out that the two arbitrary functions $\varphi$ and $\psi$ are determined by the initial conditions of the problem: $\varphi(s)+\psi(-s)=\varphi(s)-\varphi(-s)$ which represent the initial form of the curve and 
$\varphi^{\prime}(s)+\psi^{\prime}(-s)=\varphi^{\prime}(s)-\varphi^{\prime}(-s)$ the distributions of initial velocities. These conditions are of physical conditions and therefore, we cannot impose that $\varphi$ must be a defined function by means of an analytical formula because nothing can guarantee that the initial state of the string has this type of regularity. Then, Euler is obliged to assume the arbitrary function $\varphi$, a defined function, not by means of a formula, but by a graphical representation, one curve "described by the free trace of a hand". Since that the string is displaced from the equilibrium position without initial velocity, $\varphi^{\prime}$ is an even function and $\psi$ an odd one, with a period $2 a$.

Euler indicates a geometrical construction that slows for a given $t$, starting from a graphical representation of $\varphi$, the graphical representation of $y=\varphi(t+s)-\varphi(t-s)$ to be obtained. He also considers the initial form of the string as a particular one, which is given by a trigonometric form as follows:

$$
\alpha \sin (\pi s / a)+\beta \sin (2 \pi s / a)+\gamma \sin (3 \pi s / a)
$$

where the vibration is a superposition of sinusoidal modes, in growing numbers of nodes similar to what was indicated by Daniel Bernoulli for suspended strings (Euler, 1750).

For d'Alembert, Euler's solution was inacceptable due to its generality, because differential calculus cannot be applied except to well defined functions expressed by analytical formulas and not by "mechanical" curves such as the one indicated by Euler. In his 1752 work, d'Alembert returns to Brook Taylor's solution with the separation of variables in a partial differential equation. D'Alembert separated the variables in the solution and not in the equation, as we emphasized previously:

$$
\varphi(t+s)-\varphi(t-s)=\Delta(t) \Psi(s)
$$

with $\Delta$ and $\Psi$ as functions of $t$ and $s$, respectively. By applying this to the governing movement equations, we conclude that $\Delta$ and $\Psi$ must be sinusoidal.

Daniel Bernoulli entered the controversy in 1753 stating that all possible motions are mode superposition of the same type proposed by Taylor, but with any number of nodes (Bernoulli, 1755). In other words, this is the same proposed by Euler as a particular case, represented by a trigonometric series, in general. However, for Euler, an analytical expression like a trigonometric series determines a function, including its existence domain as periodic then transcendent, and cannot be, for instance, an algebraic function in the interval $[0, a]$; a solution like this is necessarily particular (Euler, 1755).

Although d'Alembert also considers this type of solution as a particular solution, in the second edition of his "Traité de Dynamique" (1758), he solved the partial differential equation of a suspended string by the separation of variables and superposition of vibration modes as follows:

$$
y=A s \cos t \sqrt{n}+B s^{\prime} \cos t \sqrt{m}+C s^{\prime \prime} \cos t \sqrt{r}+\cdots
$$

where the coefficients $s, s^{\prime}, s^{\prime \prime}$ (of $s$ only) are calculated by ordinary differential 
equations:

$$
S_{n} \mathrm{~d} S / \mathrm{d} s-(1-s) \mathrm{dd} S / \mathrm{d} s^{2}
$$

and the constants $n, m, r$ are calculated by the limit conditions $S, \mathrm{~d} S / \mathrm{d} s, S^{\prime}, \mathrm{d} S^{\prime} / \mathrm{d} s$, vanishing to $\left.s=0\right)$. Putting $t=0$, one reads:

$$
y=A S+B S^{\prime}+C S^{\prime \prime}+\cdots
$$

The young Lagrange, at that time only 23 years old, tried to reconcile part of Euler's point of view with d'Alembert's opinions. Like d'Alembert he considered that differential calculus can be applied only to functions defined by analytical formulas, but according to Euler the initial shape of the string is not expressed by this type of formula (Lagrange, 1759). He thus proposed not to use d'Alembert's partial differential equations, but to integrate the equations of motion in a discrete case (lumped mass system connected by massless strings) and to limit the obtained solutions. For the discrete case, the system of equations is as follows:

$$
\mathrm{d}^{2} y_{i} / \mathrm{d} t^{2}=y_{i+1}-2 y_{i}+y_{i-1}
$$

where $y_{i}$ is the ordinate of $m_{i}$. Lagrange introduced the functions $u_{i}=\mathrm{d} y_{i} / \mathrm{d} t$ in order to obtain a $2 n$ system of differential equations of the first order with respect to the functions $y_{i}$ and $u_{i}$. To solve this system, Lagrange made a transformation to diagonalize it, using linear combinations of the form $\sum M_{i} \mathrm{~d} y_{i} / \mathrm{d} t$ and $\sum N_{i} \mathrm{~d} u_{i} / \mathrm{d} t$, where the unknown functions $M_{i}$ and $N_{i}$ are determined considering the conditions at the extremities, being expressed by trigonometric polynomials. Using the concept of limit from $n$ to infinite masses, Lagrange transforms their finite additions into integrals, also considering that final formulas are identical to that obtained by d'Alembert and Euler with the arbitrary functions (determined by initial conditions), which could have the same generality required by Euler (Euler, 1765). It is important to emphasize that Lagrange's formulas contain the correct expressions of Fourier's coefficients of an arbitrary function, but Lagrange was reluctant to agree with this interpretation. During his life, he was persuaded that only particular and regular functions are represented by trigonometric series and this point of view would be the basis for his opposition to Fourier's work on heat theory.

The passage to limits made by Lagrange was criticized and this led to another method (Lagrange, 1762); this new method consisted of manipulating the string vibrating equation of the continuous case. Starting from the equation: $\mathrm{d}^{2} y / \mathrm{d} t^{2}=c \mathrm{~d}^{2} y / \mathrm{d} s^{2} \quad(c$ constant), multiplying by $M \mathrm{~d} s$ and integrating one obtains:

$$
\int \mathrm{d}^{2} y / \mathrm{d} t^{2} M \mathrm{~d} s=c \int \mathrm{d}^{2} y / \mathrm{d} s^{2} M \mathrm{~d} s
$$

where $M$ is a function to be determinate. A double integration by parts yields:

$$
\int \mathrm{d}^{2} y / \mathrm{d} t^{2} M \mathrm{~d} s=c\left(\mathrm{~d} y / \mathrm{d} s M-y \mathrm{~d} M / \mathrm{d} s+\int y \mathrm{~d}^{2} M / \mathrm{d} s^{2} \mathrm{~d} s\right)
$$

where the whole integrated part must vanish; this because $M$ is zero at the string 
extremities. Thus, one has:

$$
\int \mathrm{d}^{2} y / \mathrm{d} t^{2} M \mathrm{~d} s=c \int y \mathrm{~d}^{2} M / \mathrm{d} s^{2} \mathrm{~d} s
$$

Lagrange adopts the condition $\mathrm{d}^{2} M / \mathrm{d} s^{2}=k M \quad$ ( $k$ is a constant); the right-hand side becomes:

$$
c k \int M y \mathrm{~d} s=c k s
$$

the previous equation is now:

$$
\mathrm{d}^{2} S / \mathrm{d} t^{2}=c k S
$$

We thus have two ordinary differential equations to solve the calculation of $M$ and $S$; the conditions at the string extremities for an infinite series for possible values to $k, y$ is reconstructed with the corresponding values for $S$. In this method is the embryo of the idea of a "weak solution" for a partial differential equation. However, one had to wait a long time for its development; further this method led to the theory of distribution.

In 1767 Euler published a paper about the arbitrary functions that appear in the integration of partial differential equations. He showed that arbitrary functions should be taken in the more general sense, defined by curves traced freely by hand and not by functions defined by arbitrary formulas. This condition implies that the methods of analysis should be extended to these general functions. Hence, Euler's viewpoint was progressively established; in 1779, Laplace (Laplace, 1782) solved the same problem of the vibrating string using Lagrange's approach, by means of finite differences corresponding to the discretized problem and later by a passage to the limit. In other words, he adopts as a restriction of the problem that the tangents to the initial figure of the vibrating string be continuous. Yet, Condorcet (1743-1794) achieves the same conclusion using a partial differential equation of order $n$, he adopted arbitrary functions to construct the solution with the condition that their derivatives are continuous until the order $n-1$ (Condorcet, 1774).

\section{Final Remarks and Conclusion}

If we look at d'Alembert's scientific trajectory, it is possible to visualize two different but inseparable aspects. One representing a search for the rationalization of physical phenomena and its laws which frequently follow a theoretical unification based on fundamental principles of physical character. A second aspect is a critique highlighting the notions and conceptions that appear in his analyses. In addition, the privileged position that mathematics occupies. To d'Alembert among all scientific knowledge, mathematics is the nearest to pure reason because the nature of his object itself. In addition, mathematics shows the ways to achieve sureness with respect to reasoning (Paty, 1998).

The theory of partial differential equations appears with d'Alembert as a new branch of differential and integral calculus; it solved many problems of continuum mechanics, with d'Alembert aware that he had created a new discipline 
(Paty, 2001). He elaborated the initial studies and methods concerned with the integrability conditions of the differential forms; the skill and originality of his trajectory through calculus is remarkable. His study of the linear variation of variables contains the idea of characteristics, which is a technique for solving partial differential equations. It usually applies to first order differential equations, although from a more general point of view the characteristic method is valid for any hyperbolic partial differential equation. The method consists of reducing a partial differential equation to a family of ordinary differential equations over which the solution can be integrated from some initial data.

D'Alembert discovers the introduction of arbitrary functions in the solution of equations initiating a famous controversy involving the concept and the domain of a function as a direct application to differential and integral calculus. He proposes the method of separating variables which would be explored by Fourier in his theory of heat.

The main contribution to the study of wave equation was made by d'Alembert, with important developments by Daniel Bernoulli, Lagrange, Laplace and others, referred in this paper. The context of the controversy about the solution of the vibrating string made many important contributions to appear, enriching the subject. In addition, it is fundamental to emphasize the achievements made by Euler. He introduces new ideas to the first order equations, showing that these equations are not necessarily linear; he explores Legendre's transformation and prepares the route for the notion of contact transformation (Nielsen, 2010).

Therefore, a deep understanding of d'Alembert and Euler's methods cannot be achieved without a geometric interpretation of the theory and remaining only in the plane of pure analytical manipulations. These geometric interpretations have become very strong in the present days.

\section{Conflicts of Interest}

The author declares no conflicts of interest regarding the publication of this paper.

\section{References}

Bernoulli, D. (1740). Theoremata de oscillationibus corporum filo flexibili connexorum et catenae verticaliter suspensae. Comm. acad. sc. Petrop., 6(1732-33), 108-122.

Bernoulli, D. (1755). Reflexions et éclaircissements sur les nouvelles vibrations des cordes exposées dans les mémoires de l'académie de 1747 et 1748. Hist. Acad. sc., Berlin, 9(1753), 147-172.

Condorcet, de C. (1774). Sur la détermination des fonctions arbitraires qui entrent dans les intégrales des équations aux différences partièlles. Hist. Acad. Sc., Paris, (1771), 49-74.

D’Alembert, J. Le R. (1743). Traité de dynamique. Gauthier-Villars et Cie. Éditions.

D’Alembert, J. Le R. (1747). Réflexions sur la cause generale des vents. David, Paris.

D’Alembert, J. Le R. (1750). Recherches sur la courbe que forme une courbe tendue, mise 
en vibration. Hist. Acad. sc. Berlin, 3(1747), 214-219, 220-229.

Euler, L. (1749). De vibratione chordarum execitatio. Nova acta erud., 512-527.

Euler, L. (1750). De vibratione chordarum execitatio. Nova acta erud., 1750, 512-527.

Euler, L. (1755). Remarques sur les mémoires précédents de M. Bernoulli. ibd., 9(1753), 196-222.

Euler, L. (1765). De usu functionum discontinuarum in analysi. Novi Comm. Acad. Sc., Petrop. II, 67-102 = Op. Omn. $\mathrm{I}_{23}, 74-87$.

Euler, L. (1980). Opera omnia, Series 4A, Vol. 5 (A. P. Juskevik \& R. Taton, Eds.). Basel: Birhäuse.

Fourier, J. (1822). Théorie analytique de la chaleur. Paris: Éditions Jacques Gabay.

Knobloch, E. (2018). Euler and d'Alembert-Brothers Only in Mind. Acta Baltica Historiae et Philosophiae Scientiarum, 6, 106-126.

https://doi.org/10.11590/abhps.2018.2.06

Lagrange, J. L. (1759). Recherches sur la nature et la propagation du son. Misc. Taurin., I3, I-X, I-112.

Lagrange, J. L. (1762). Nouvelles recherches sur la nature et la propagation du son. ibid., II2 (1760-61), II-172.

Laplace, P. S. (1782). Mémoire sur les suites. Hist. Acad. Sc. Paris, (1779), 207-309 (1782), Oeuvres 10, 1-89.

Nielsen, F. (2010). Legendre Transformation and Information Geometry. Paris: Sony Computer Science Laboratories, Inc.

Oliveira, A. R. E. (2007). Euler's Contribution to Classical Mechanics. 12th IFToMM World Congress, Besançon, 18-21 June 2007

Paty, M. (1993). D'Alembert, Jean Le Rond (1717-1783), Dictionaire des philosophes, déuxième éd. Rev. et augm. Paris: Presses Universitaires de France.

Paty, M. (1998). D’Alembert ou la raison physico-mathématique au siècle des Lumières. Paris: Societé d'Édition Les Belles Lettres.

Paty, M. (2001). D’Alembert, la science newtoniènne et l'héritage cartesièn. Corpus (Revue de philosophie. Corpus des oeuvres de philosophie en langue française), No. 3, 19-64.

Paty, M. (2002). Analyse et Dynamique: Études sur l'oeuvre de d'Alembert. Quebec: Les Presses de l'Université de Laval. 